\title{
Precocious quantitative cognition in monkeys
}

\author{
Stephen Ferrigno $^{1,2}$ • Kelly D. Hughes ${ }^{1,2}$ • Jessica F. Cantlon ${ }^{1,2}$
}

Published online: 18 July 2015

(C) Psychonomic Society, Inc. 2015

\begin{abstract}
Basic quantitative abilities are thought to have an innate basis in humans partly because the ability to discriminate quantities emerges early in child development. If humans and nonhuman primates share this developmentally primitive foundation of quantitative reasoning, then this ability should be present early in development across species and should emerge earlier in monkeys than in humans because monkeys mature faster than humans. We report that monkeys spontaneously make accurate quantity choices by 1 year of age in a task that human children begin to perform only at 2.5 to 3 years of age. Additionally, we report that the quantitative sensitivity of infant monkeys is equal to that of the adult animals in their group and that rates of learning do not differ between infant and adult animals. This novel evidence of precocious quantitative reasoning in infant monkeys suggests that human quantitative reasoning shares its early developing foundation with other primates. The data further suggest that early developing components of primate quantitative reasoning are constrained by maturational factors related to genetic development as opposed to learning experience alone.
\end{abstract}

Keywords Numerosity $\cdot$ Numerical cognition $\cdot$ Animal cognition $\cdot$ Cognitive development

Cognitive research with infant and juvenile monkeys is important for theories of human development because it can help

Jessica F. Cantlon

jcantlon@rcbi.rochester.edu

1 Department of Brain and Cognitive Sciences, University of Rochester, Meliora Hall Box 270268, Rochester, NY 14627, USA

2 Seneca Park Zoo, Rochester, NY 14621, USA to reveal the evolutionary foundation of human reasoning and the role of genetic and maturational factors in cognitive development (Bjorklund \& Pellegrini, 2002; Diamond, 1990, 1991; Gómez, 2005; Rosati, Wobber, Hughes, \& Santos, 2014). Current theories of human mathematics development argue that the ability to quantify sets of objects nonverbally is an innate component of human cognition (Feigenson, Dehaene, \& Spelke, 2004; Gallistel, 1990; Gallistel \& Gelman, 2000; Gelman, 1972; Spelke, 2011). If the ability to quantify is part of our evolutionary endowment, then that ability should be present in our phylogenetic relatives (other primates). Substantial evidence has shown that this is the case (Beran, 2008; Brannon, 2005; Cantlon, 2012; Nieder \& Dehaene, 2009). Many studies with a variety of different species have shown that nonhuman primates can match stimuli, such as sets of objects or visual arrays, based on quantity and can determine the larger or smaller of a selection of sets. An open question, however, is whether quantitative reasoning develops early in nonhuman primate development, as it does in humans.

Here we test whether experiment-naïve 1-year-old infant monkeys spontaneously make quantitative judgments between sets of objects. If quantitative reasoning has an innate basis in nonhuman primates, then it should emerge early in development. In fact, if there is an innate basis of quantitative reasoning, then monkeys should develop quantitative reasoning capabilities even earlier in development than human children because they mature faster than humans.

Although implicit measures of cognition, such as patterns of looking, have revealed quantitative sensitivities in human infants within the first year of life (Cordes \& Brannon, 2008; Izard, Sann, Spelke, \& Streri, 2009; Xu \& Spelke, 2000), research that uses explicit judgment tasks indicates that human children do not make reliable quantity judgments for sets larger than three items until 2.5 to 3 years of age (Brannon \& Van de Walle, 2001; Feigenson \& Carey, 2003; Feigenson, Carey, 
\& Hauser, 2002; Mix, Huttenlocher, \& Levine, 2002; Sella, Berteletti, Lucangeli, \& Zorzi, 2015). This is a puzzling finding in child development because children are capable of perceiving, reaching, grasping, and making choices among objects well before this age, but, yet, they do not use those capabilities to make explicit judgments of quantities. It is currently unclear to what degree children's failures to make quantitative judgments are explained by the pace of their genetic maturation and/or inadequate learning experience (Gelman, 1972; Mehler \& Bever, 1967; Mix, 2002).

Research with young monkeys could shed some light on this issue. In the domain of physical growth and motor behavior, monkeys mature much faster than humans. For example, human infants begin to crawl when they are 9-10 months of age, whereas monkey infants can crawl within 1 month of birth (Hinde \& Spencer-Booth, 1967). Perceptual development in monkeys is also accelerated compared to humans. Monkey infants develop the ability to locate occluded objects by 4 months of age, which is three times faster than human infants (Diamond, 1990, 1991; Gómez, 2005). Thus, it appears that initial object-tracking abilities are yoked to the rate of physical development in primates. Based on these prior data, we reasoned that quantitative development in primates might also be largely constrained by the unfolding of genetically predetermined abilities, or maturation, in which case monkey infants should engage in quantitative judgments at an earlier point in development than human infants. We also measured the role of learning in infant monkey quantity development by comparing the performance of infant monkeys to that of adult animals from their group, as well as rates of task learning between infant and adult animals. If learning plays a critical role in the spontaneous quantitative reasoning of monkeys, then infants should perform worse than adults, and learning rates should differ between age groups.

\section{Method}

\section{Subjects}

Eight experiment-naïve adult (adults: mean $=8$ years, range 5-14 years), and two infant olive baboons (Papio anubis; infants: mean $=18$ months, range $17-18$ months) participated in the study. Baboons are infants until 1.5 to 2 years of age, when they transition to being juveniles (http://www.princeton. edu/ baboon/cradle to grave.html). The attributes that distinguish infant baboons from juveniles is that they have darker fur and more facial wrinkles than older animals, and suckle and ride on their mothers (Altmann, Altmann, Hausfater, \& McCuskey, 1977). The subjects are described as experiment naïve because they had never participated in any quantity task prior to this experiment. All animals were part of the same social group and were tested and housed at the Seneca Park Zoo in Rochester, NY. Animals received primate chow, fruits, and vegetables every morning, and water was available ad libitum. All procedures were approved by the Seneca Park Zoo Research Committee.

\section{Apparatus}

The apparatus consisted of a short rectangular table $(75-\mathrm{cm}$ long $\times 35-\mathrm{cm}$ deep $\times 17-\mathrm{cm}$ high) that was a comfortable height for a seated baboon. The front of the apparatus was shielded Plexiglas and had three equally spaced ports for subjects to indicate their choice. Experimental manipulations were conducted on a sliding panel $(75-\mathrm{cm}$ long $\times 17-\mathrm{cm}$ deep) that sat atop the table. When the sliding panel was pushed forward, subjects could reach through a port in the Plexiglas and indicate their choice. Three identical, opaque, cardstock cylinders were placed on the sliding board in front of three corresponding ports. Once items were dropped into the cylinders, the items were hidden from the subject. The items to be enumerated were shelled half peanuts.

\section{Procedure}

Animals were presented with homogenous sets of food items in a quantity choice task in order to keep the task as natural and intuitive for the animals as possible - quantitative reasoning in humans and nonhuman animals involves complex interactions between numerical, spatial, and temporal representations, and animals most likely use a combination of these cues to judge quantities in nature (Gallistel, 1990). A trial was initiated when the subject was seated at and attentive to the apparatus. Subjects were presented with two sets of food items, ranging in quantity from 1 to 8 items. The sets were presented simultaneously with one set of food items in the palm of each hand and then placed into two opaque containers. Although there were always three cylinders on the board, only two of these cylinders were baited with food on each trial. The presence of the third cylinder allowed us to monitor subjects' understanding of the general task requirement that only baited cups should be chosen. Subjects almost never selected the empty cylinder $(<1 \%$ of trials) indicating that that understood and attended to the task.

After the cylinders had been baited with peanuts, the panel was pushed forward and the subject was allowed to choose one food cache by touching the port in front of it. The experimenter fixed their gaze on the floor as the subject made their choice. The subject was given the contents of the chosen cache. Since the subjects were rewarded with some quantity of food on every trial, they were nondifferentially reinforced and thus were not conditioned to make accurate quantity judgments. This feature of the design permitted us to measure animals' spontaneous quantitative sensitivity over many trials. Sessions were recorded and coded for accuracy offline. Individuals were tested between one and 
three times a week. This procedure was used throughout the training and testing phases.

\section{Training}

In order to familiarize subjects with the testing procedure, we initially exposed them to only a single numerical comparison ( 1 vs. 2 for adult animals and 1 vs. 3 for infant animals). Sessions consisted of 24-36 trials. Subjects were trained until they chose the larger set at above chance levels within a single session (adult threshold $=24 / 36$ correct, infant threshold $=16 /$ 24 correct). During this initial familiarization phase, which was the first ever experiment in which subjects participated, infants required an average of 90 trials to reach criterion, and adults required an average of 55 trials. Trials were counterbalanced for baiting locations. Progress through the session was closely monitored. If a gap of 5 min occurred between two trials due to subject inattention, the session was terminated, and training resumed the next time the subject was available. Terminated sessions were rare and excluded from analyses. Once the subject passed the training criterion they began the testing phase of the experiment.

\section{Testing}

Testing was conducted over multiple 30 -min sessions. All number pairs ranging from 1 to 8 items were tested. The beginning of each testing session consisted of 4-6 warm-up trials using the training number pairs ( 1 vs. 2 for adults and 1 vs. 3 for infants). If the subject failed half of these first trials, testing with that subject was terminated for the day. Terminated sessions due to poor performance were rare $(8 \%)$ and were excluded from analyses. The order of the test trials was randomized within and between subjects. Also, as in training, baiting locations were randomized. If a gap of 5 min occurred between two trials due to subject inattention, the session was stopped, and the remaining trials were resumed after a warmup during the next testing day.

\section{Experimenter cueing control condition}

Experimenter cueing is important to control because some have argued that animals can use subconscious human cues to solve cognitive tasks, as in the Clever Hans phenomenon (e.g., Beran, 2012; Hediger, 1981). Several experimental design features make this an unlikely possibility in the current experiment. First, animals were rewarded on every trial, regardless of whether or not they choose the larger set. Subjects who could not discriminate the quantities of the two sets could not learn to use associative cues under these conditions of constant reinforcement. Second, as will become clear in the Results section, we can conduct statistical analyses to determine whether the animals' responses are accounted for by variation in the quantitative values that were presented which would not be predicted under an associative cueing account of their behavior. Third, experimenters obscured their gaze upon presentation of the choice options to the subjects. In addition to these experimental design features, we also conducted a separate cueing control condition with a subset of the subjects to rule out the possibility that human cues were used routinely by the animals during the task ( $N=2$ adults; see also Barnard et al., 2013; Cantlon et al., 2015). On each trial, each of two different experimenters baited one of the two cylinders. The experimenters were blind to the quantity of food in the other set and thus had no information about which cylinder was correct. This eliminates the possibility of subconscious cueing of the animals. Animals were initially tested on approximately 55 trials using a subset of the number pairs (1 vs. 2 and 2 vs. 9) and then were tested on 100 additional trials over a broader range of values from 1 to 8 . Items were baited sequentially. This condition was tested after the main experiment over two to three sessions. If the animals used human cues to solve the task in the main experiment, their performance should fall to chance when those cues were neutralized in this control condition.

\section{Results and discussion}

Infant animals spontaneously chose the larger quantity on a majority of the trials (Accuracy: $74 \%, 156 / 210, p<.001$ ). We tested whether infants' accuracy was modulated by the quantitative values that they compared. The key behavioral signature of nonverbal quantitative processing is the ratio effect, wherein accuracy decreases as quantities become larger and closer in value (i.e., as the ratio between quantities narrows; Gallistel \& Gelman, 2000). This principle is also known as Weber's law of psychophysics. The ratio effect is a signature of analog magnitude representation wherein quantities are represented nonverbally as noisy approximations of their objective values as opposed to digital, precise values, as is done with symbolic counting (Gallistel, 1990; Gallistel \& Gelman, 2000; Feigenson et al., 2004). Several prior studies have reported ratio effects in the quantity judgments of adult monkeys (e.g., Barnard et al., 2013; Beran, 2007; Cantlon \& Brannon, 2006; Cantlon et al., 2015). We found that infant monkeys' performance was modulated by the ratio between the two caches. Accuracy decreased as the ratio between quantities (smaller value/ larger value) increased $(R=-0.71, p<.05)$. This finding indicates that infant monkeys used analog quantitative reasoning to solve the task. This is evidence that nonverbal quantitative reasoning emerges spontaneously and early in nonhuman primate development, by at least 1 year of age.

Experiment-naïve adult animals from the same social group as the infants were tested on the same quantity choice 
task $(N=8$, mean age $=8$ years $)$. The adult monkeys performed significantly above chance across trials, and, like infants, their performance was modulated by the ratio between the quantities of the choices (Accuracy: $73 \%, 400 / 546, p<$ $.001 ; R=-0.88, p<.01)$. Monkeys also performed significantly above chance from the first session of the cueing control condition where human experimenters were blind to the relative values of the two choice options (Mean Accuracy Overall: $78 \%$; Binomial tests on first session; Monkey 1: 19/24, $p<.01$; Monkey 2: 17/24, $p<.05$ ). There was no decrease in performance on the cueing control condition compared to the main experiment (Main Experiment: $74 \%$, Control Condition: $\left.78 \% ; X^{2}(1, N=1,049)=2.3849, p=.12\right)$. The significant effect of ratio on monkeys' performance combined with the lack of a decrement in performance on the control condition indicates that monkeys compared the quantities of the sets rather than using an alternative strategy.

Next, we compared performance between infant and adult monkeys to examine the role of experience in their spontaneous quantitative reasoning. For this analysis, we measured monkeys' Weber fractions, which is the proportion difference between quantities that a subject requires to discriminate them reliably ( $\sim 75 \%$ accuracy). We determined subjects’ Weber fractions by finding the best fit between their accuracy on the different quantity pairs that were tested and the predicted accuracy of a model of Weber's law that tested a range of possible Weber fractions (Pica, Lemer, Izard, \& Dehaene, 2004). A smaller Weber fraction (w) reflects better discrimination sensitivity, such that a $w$ of 1 represents the ability to discriminate sets with a 1:2 ratio and a $w$ of .5 represents the ability to discriminate sets with a $2: 3$ ratio. The results revealed that adults and infants had similar weber fractions of around .7 (Adult $w=.75$, Infant $w=.71$; Goodness-of-fit Adults: $R^{2}=.76, p<.01$, Infants: $\left.R^{2}=.50, p<.05\right)$. The nearest whole number fraction that can be reliably discriminated at this Weber fraction is 3:5, which is a similar sensitivity to 3-year-old human children who are reported to perform at slightly worse than a 2:3 ratio limit in comparable tasks with similar measurement methods (3-year-old children: $w=.53$; Halberda \& Feigenson, 2008).

A statistical comparison of adult and infant accuracy and Weber fractions revealed similar quantitative sensitivity for the infant and adult animals (see Fig. 1; Group Comparison: $\left.X^{2}(1, N=756)=.082, p=.78\right)$. The finding of no difference between infant and adult animals' Weber fractions also held using Crawford's modified $t$ statistic, a statistic that is used in neuropsychological case studies to determine whether an individual's performance significantly differs from a reference group (Crawford \& Howell, 1998; Infant 1 vs. Adults: $t=.73$, $p=.53$; Infant 2 vs. Adults: $t=-.50, p=.64$ ). We also compared the standard deviations of monkeys' performance using a linear regression with predictors of Age Group and Ratio over the standard deviation of each subject's accuracy across sessions for each quantity ratio. We found no significant effect of Age Group $\left(\beta_{\text {age }}=-.02, p=.66\right)$ nor an interaction of Age Group by Ratio $\left(\beta_{\text {age*ratio }}=.20, p=.26\right)$ on the standard deviation of accuracy. This indicates that infants were not significantly more variable than adults in their quantity judgments. Finally, the quantitative sensitivity of our adult and infant animals fell within the interquartile range of that observed by other labs, in previous experiments with untrained monkeys (Jones et al., 2014). This shows that the animals in the current experiment were typical of their population. Thus, infant monkeys performed as well as typical adult monkeys in their quantity discriminations. This finding indicates that the spontaneous quantitative cognition of nonhuman primates is relatively stable after 1 year of development.

In light of evidence that human children less than 2 years of age have difficulty comparing quantities of greater than three items during choice tasks (Feigenson \& Carey, 2003; Sella et al., 2015), we explored whether infant monkeys exhibit similar difficulties with large quantities. We tested whether monkeys performed better on trials in which both sets contained only small numbers of items $(<4)$. Accuracy on small sets was highly similar to accuracy on trials in which one or more of the sets contained five or more items (Adults: small number accuracy $73 \%$, large number accuracy $73 \%$; Infants: small number accuracy $74 \%$, large number accuracy $74 \% ; t(9)=.27, p=.79)$. It also has been reported that young human children fail to compare sets that span small and large quantities, such as 3 versus 6 (Feigenson et al., 2002). In contrast, infant and adult monkeys performed significantly above chance on the span trials (Adult accuracy: $75 \%, 123 /$ 163, $p<.001$; Infant accuracy: $86 \%, 49 / 57, p<.001)$. This suggests that, unlike 1-year-old human children, 1-year-old monkeys are capable of making adaptive choices over a large range of quantities. This is further evidence that quantitative cognition matures faster in nonhuman primates than in humans.

To test for effects of experience, we calculated the statistical effects of age and ratio on accuracy using logistic regression. If experience affects accuracy or modulates accuracy as a function of the ratio between the sets, we should see either a main effect of Age or an interaction between Age and Ratio. We found neither a main effect of Age $\left(\beta_{\text {age }}=-.01, p=.88\right)$ nor an interaction of Age by Ratio ( $\beta_{\text {ratio*age }}=-.09, p=.29$ ). Instead, we found a main effect of ratio on subjects' accuracy that was independent of age $\left(\beta_{\text {ratio }}=-.37, p<.001\right)$, indicating that Weber's law predicts accuracy equivalently for infant and adult monkeys. This analysis confirms that infant and adult animals have equivalent quantitative abilities.

Next, we examined the effect of learning by including trial number and the interaction of trial number by age as predictor variables in our logistic regression. If experience influences performance, then we should see a positive effect of Trial Number on performance. Alternatively, if there are differences 

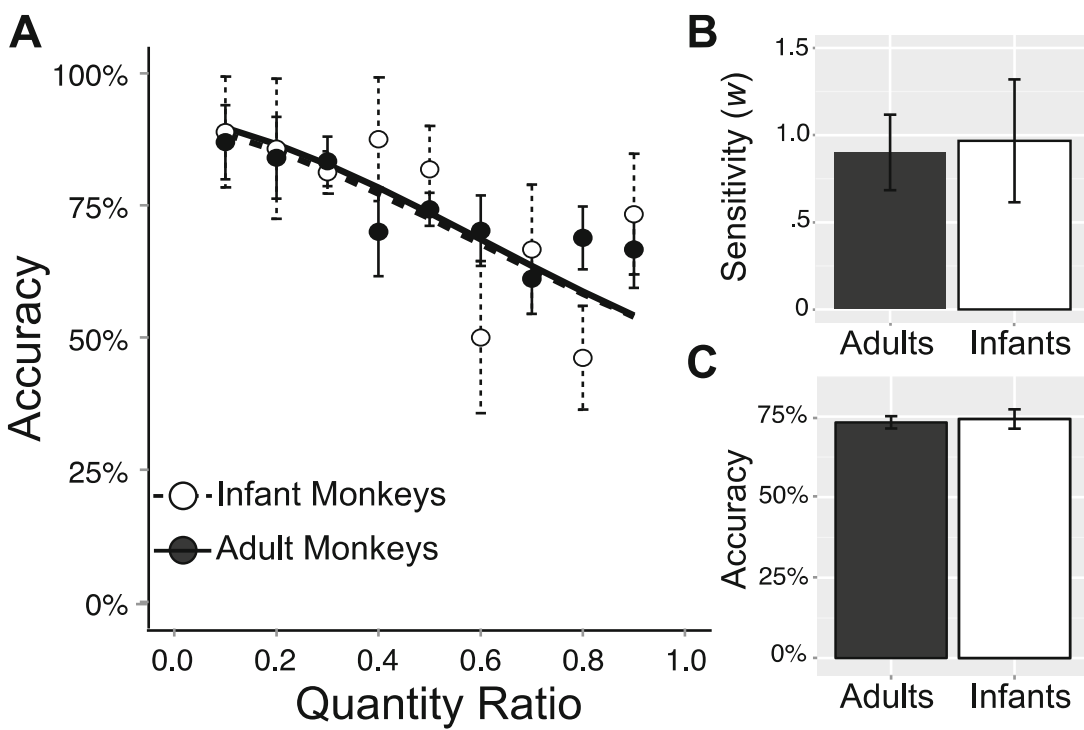

Fig. 1 (A) Adult and infant monkeys' accuracy as a function of quantity ratio (smaller quantity/larger quantity). Solid lines (adults) and dotted lines (infants) represent fits predicted by a model of Weber's law (following Cantlon \& Brannon, 2007; Pica et al., 2004; Goodness-offit: Adults: $R^{2}=.76, p<.01$, Infants: $R^{2}=.50, p<.05$ ). Error bars represent the standard error of the mean. (B) Average Weber fractions (w) for monkeys for whom individual Weber fractions could be calculated

in how experience affects infants' versus adults' performance, we should see a significant interaction between Trial Number and Age. Our results show that performance slightly improved over testing $\left(\beta_{\text {trial }}=.49, p<.001\right)$, but there was no difference in the degree of improvement between adult and infant animals $\left(\beta_{\text {age*trials }}=.12, p=.30\right)$. This finding shows that experience making quantity discriminations improves performance at the same rate for infant and adult animals, and thus developmental learning is not more rapid than adult learning. This means that our findings of equivalent quantitative cognition in infant and adult animals cannot be explained by rapid early learning in infant animals.

\section{Conclusion}

Our data from nonhuman primates provide new insights into a number of issues that bear on human cognitive development. First, the data we report here are the first to show that spontaneous quantitative reasoning emerges within 1 year of age in nonhuman primates. We hypothesized that if quantitative reasoning has a common developmental basis in human and nonhuman primates then it should emerge early in development across primate species. Our finding that 1-year-old monkeys spontaneously judge quantitative values confirms that quantitative reasoning is an early developing cognitive ability in nonhuman primates as in humans (e.g., Cordes \& Brannon, 2008; Spelke, 2011; Xu \& Spelke, 2000). These findings
(6/8 adults and all infants). The Weber fraction measures sensitivity to quantity, with smaller values indicating better acuity. A $w$ of 0.5 represents the ability to discriminate sets with a $2: 3$ ratio approximately $75 \%$ of the time. Error bars represent the standard error of the mean. (C) Adult and infant monkeys' overall accuracy. Error bars represent the standard error of the mean.

provide new evidence of a common developmental foundation for quantitative cognition in humans and nonhuman primates.

We also hypothesized that if the ability to make quantitative judgments is constrained by the unfolding of genetically predetermined abilities, or maturation, then monkeys should develop the ability to make quantitative judgments earlier than humans because they mature faster. This type of phenomenon has been shown previously in studies comparing the performance of monkeys and humans for lower level abilities such as object representation (Diamond, 1990, 1991; Gómez, 2005). In those prior studies it was shown, for example, that infant monkeys develop the ability to detect and reach for objects at an earlier point in development compared to humans, indicating that the development of object perception is affected by the species' rate of growth. Our data provide the first evidence that primate quantitative cognition also is affected by a species rate of growth as monkeys develop the capacity for explicit quantitative judgments more rapidly than humans - 1-year-old monkeys are capable of making explicit quantity judgments that human children fail to express until 2.5 or 3 years of age (Brannon \& Van de Walle, 2001; Cantlon, Safford, \& Brannon, 2010; Feigenson \& Carey, 2003; Sella et al., 2015). Further testing with younger animals is needed to determine the exact age at which this capacity for adult-like explicit quantitative judgments emerges. The fact that primate quantitative development is rapid, like the pace of their genetic growth, implicates maturational factors as an important constraint on quantitative development. 
Finally, we hypothesized that if learning experience is the primary factor in the spontaneous quantitative reasoning of monkeys, then infant animals should perform worse than adult animals and learning rates should differ between age groups. Although both infant and adult monkeys showed improvement in performance over testing, indicating that quantitative learning can occur in infant and adult animals, there was no effect of age on monkeys' spontaneous quantity abilities and there were no differences in learning rates between age groups. It is not likely that rapid learning explains the rapid development of quantitative reasoning in infant monkeys since there was nothing different about the learning of infant monkeys compared to adult monkeys. We argue that learning experience is not the primary factor in the spontaneous quantitative abilities of monkeys. Instead, we argue that primate quantitative cognition initially emerges from the same kinds of maturational gene-environment interactions that result in basic perceptual processes.

The argument that maturation is an important factor in quantitative development does not imply that environmental input is unimportant. For example, in the case of object detection, animals raised in impoverished environments are unlikely to develop normal object perception abilities (Diamond, 1990, 1991). The rapid genetic maturation of monkeys is not deterministic of cognitive abilities but rather allows them to access key environmental inputs for developing those abilities at an earlier time than humans. Our data provide novel evidence that maturation is a factor in quantitative development, which includes the species' genetic physical development as well as the access to environmental inputs afforded by that physical development. The specific genetic events and environmental inputs that are necessary for quantitative reasoning to emerge are not yet known. However, our finding that quantitative cognition emerges within 1 year in monkeys suggests that the necessary precursors are relatively fundamental features of the environment.

Taken together, our results implicate the existence of evolutionarily primitive quantitative abilities across primate species that are critically influenced by the species' rate of maturation. These results add a new type data, developmental data from nonhuman primates, to the growing literature implicating a fundamental link between the cognitive abilities of humans and nonhuman primates in the domain of quantitative cognition (Beran, 2008; Brannon, 2005; Cantlon, 2012; Cantlon \& Li, 2013; Nieder \& Dehaene, 2009; Spelke, 2011).

Acknowledgements We thank Alyssa Arre, Allison Barnard, Elizabeth Brown, Louis DiVincenti, Sarah Koopman, Celia Litovsky, and Sabina Noll for assistance with testing subjects and coding data. We also thank Jeff Wyatt and the Seneca Park Zoo for supporting cognitive research with their animals. This work is supported by the National Science Foundation (DRL1459625), James S. McDonnell Foundation (220020300), and Alfred P. Sloan Foundation (BR2013-019-A) to J. F. Cantlon.

\section{References}

Altmann, J., Altmann, S. A., Hausfater, G., \& McCuskey, S. A. (1977). Life history of yellow baboons: Physical development, reproductive parameters, and infant mortality. Primates, 18(2), 315-330.

Barnard, A. M., Hughes, K. D., Gerhardt, R. R., DiVincenti, L., Bovee, J. M., \& Cantlon, J. F. (2013). Inherently analog quantity representations in olive baboons. Frontiers in Psychology, 4(253), 1-11.

Beran, M. J. (2007). Rhesus monkeys (Macaca mulatta) enumerate large and small sequentially presented sets of items using analog numerical representations. Journal of Experimental Psychology: Animal Behavior Processes, 33(1), 42.

Beran, M. J. (2008). The evolutionary and developmental foundations of mathematics. PLoS Biology, 6(2), e19.

Beran, M. J. (2012). Did you ever hear the one about the horse that could count? Frontiers in Psychology, 3, 357.

Bjorklund, D. F., \& Pellegrini, A. D. (2002). The origins of human nature: Evolutionary developmental psychology. Washington, DC: American Psychological Association.

Brannon, E. M. (2005). What animals know about numbers. In J. I. D. Campbell (Ed.), Handbook of mathematical cognition (pp. 85-107). New York, NY: Psychology Press.

Brannon, E. M., \& Van de Walle, G. A. (2001). The development of ordinal numerical competence in young children. Cognitive Psychology, 43(1), 53-81.

Cantlon, J. F. (2012). Math, monkeys, and the developing brain. Proceedings of the National Academy of Sciences, 109(Suppl. 1), 10725-10732.

Cantlon, J. F., \& Brannon, E. M. (2006). Shared system for ordering small and large numbers in monkeys and humans. Psychological Science, 17(5), 401-406.

Cantlon, J. F., \& Brannon, E. M. (2007). Basic math in monkeys and college students. PLoS Biology, 5(12), e328.

Cantlon, J. F., \& Li, R. (2013). Neural activity during natural viewing of Sesame Street statistically predicts test scores in early childhood. PLoS Biology, 11(1), e1001462.

Cantlon, J. F., Piantadosi, S., Ferrigno, S., Hughes, K., \& Barnard, A. (2015). The origins of counting algorithms. Psychological Science, 26(6), 853-865.

Cantlon, J. F., Safford, K. E., \& Brannon, E. M. (2010). Spontaneous analog number representations in 3 -year-old children. Developmental Science, 13(2), 289-297.

Cordes, S., \& Brannon, E. M. (2008). Quantitative competencies in infancy. Developmental Science, 11(6), 803-808.

Crawford, J. R., \& Howell, D. C. (1998). Comparing an individual's test score against norms derived from small samples. The Clinical Neuropsychologist, 12(4), 482-486.

Diamond, A. (1990). The development and neural bases of memory functions, as indexed by the A-not-B and delayed response tasks, in human infants and infant monkeys. Annals of the New York Academy of Sciences, 608, 267-317.

Diamond, A. (1991). Frontal lobe involvement in cognitive changes during the first year of life. In K. R. Gibson \& A. C. Petersen (Eds.), Brain maturation and cognitive development: Comparative and cross-cultural perspectives (pp. 127-180). Hawthorne, NY: Aldine de Gruyter.

Feigenson, L., \& Carey, S. (2003). Tracking individuals via object-files: Evidence from infants' manual search. Developmental Science, 6(5), 568-584.

Feigenson, L., Carey, S., \& Hauser, M. (2002). The representations underlying infants' choice of more: Object files versus analog magnitudes. Psychological Science, 13(2), 150-156.

Feigenson, L., Dehaene, S., \& Spelke, E. (2004). Core systems of number. Trends in Cognitive Sciences, 8(7), 307-314. 
Gallistel, C. R. (1990). The organization of learning. Cambridge, MA: MIT press.

Gallistel, C. R., \& Gelman, R. (2000). Non-verbal numerical cognition: From reals to integers. Trends in Cognitive Sciences, 4(2), 59-65.

Gelman, R. (1972). Logical capacity of very young children: Number invariance rules. Child Development, 43(1), 75-90.

Gómez, J. C. (2005). Species comparative studies and cognitive development. Trends in Cognitive Sciences, 9(3), 118-125.

Halberda, J., \& Feigenson, L. (2008). Developmental change in the acuity of the "Number Sense": The Approximate Number System in 3-, 4-, 5-, and 6-year-olds and adults. Developmental Psychology, 44(5), 1457.

Hediger, H. K. (1981). The Clever Hans phenomenon from an animal psychologist's point of view. Annals of the New York Academy of Sciences, 364(1), 1-17.

Hinde, R. A., \& Spencer-Booth, Y. (1967). The behaviour of socially living rhesus monkeys in their first two and a half years. Animal Behaviour, 15(1), 169-196.

Izard, V., Sann, C., Spelke, E. S., \& Streri, A. (2009). Newborn infants perceive abstract numbers. Proceedings of the National Academy of Sciences, 106(25), 10382-10385.

Jones, S. M., Pearson, J., DeWind, N. K., Paulsen, D., Tenekedjieva, A. M., \& Brannon, E. M. (2014). Lemurs and macaques show similar numerical sensitivity. Animal Cognition, 17(3), 503-515.
Mehler, J., \& Bever, T. G. (1967). Cognitive capacity of very young children. Science, 158(3797), 141-142.

Mix, K. S. (2002). The construction of number concepts. Cognitive Development, 17(3), 1345-1363.

Mix, K. S., Huttenlocher, J., \& Levine, S. C. (2002). Quantitative development in infancy and early childhood. New York, NY: Oxford University Press.

Nieder, A., \& Dehaene, S. (2009). Representation of number in the brain. Annual Review of Neuroscience, 32, 185-208.

Pica, P., Lemer, C., Izard, V., \& Dehaene, S. (2004). Exact and approximate arithmetic in an Amazonian indigene group. Science, 306(5695), 499-503.

Rosati, A. G., Wobber, V., Hughes, K., \& Santos, L. R. (2014). Comparative developmental psychology: How is human cognitive development unique? Evolutionary Psychology, 12(2), 448-473.

Sella, F., Berteletti, I., Lucangeli, D., \& Zorzi, M. (2015). Spontaneous non-verbal counting in toddlers. Developmental Science. doi:10. 1111/desc. 12299

Spelke, E. S. (2011). Natural number and natural geometry. In D. Stanislas \& E. Brannon (Eds.), Space, time and number in the brain: Searching for the foundations of mathematical thought (pp. 287317). London: Academic Press.

Xu, F., \& Spelke, E. S. (2000). Large number discrimination in 6-monthold infants. Cognition, 74(1), B1-B11. 